\title{
Article
}

\section{Dementia Patient's Meal Monitoring Systems Using Weight and Temperature Sensors}

\author{
Ji-Eun Joo ${ }^{1,2}$, Haewon Hwang ${ }^{1}$, Yujin Jeon ${ }^{1}$, Jaewon Jung ${ }^{1}$, Yu Hu 1,2, and Sung Min Park 1,2,* \\ 1 Department of Electronic and Electrical Eng., Ewha Womans University, Seoul, Korea \\ 2 Graduate Program in Smart Factory, Ewha Womans University, Seoul, Korea \\ * Correspondence: smpark@ewha.ac.kr
}

\begin{abstract}
This paper presents a couple of meal monitoring systems for senile dementia patients by using electronic weight and temperature sensors. These monitoring systems enable to convey the information of the amount of meal taken by the patients in real-time via wireless communication networks onto the mobile phones of their families or nurses in charge. Thereby, the nurses can easily spot the most desperate patient to take care of while the families can have relief to see the crucial information for survival of their parents at least three times a day. Meanwhile, the senile dementia patients tend to suffer the burn of their tongues because they can hardly recognize the temperature of hot meals served and therefore avoid the burn of tongues. This phenomenon can be discarded by utilizing the meal temperature monitoring system which displays alarm to the patients when the meal temperature is above the reference. These meal monitoring systems can be easily implemented by utilizing low-cost sensor chips and Arduino UNO boards so that elder-care hospitals and nursing homes can afford to exploit them with no additional cost. Hence, we believe that the proposed monitoring systems would be a potential solution to provide a great help and relief not only for the professional nursing nurses working in elder-care hospitals and nursing homes, but also for the families of the dementia patients.
\end{abstract}

Keywords: Arduino; Bluetooth; load cell; monitoring system; temperature sensor; weight sensor

\section{Introduction}

Recently, aging societies have become a global issue which is particularly serious in Far-East owing to the life longevity benefitted from their advanced health-care systems along with the economic growth for the past decades. However, these aging societies naturally and inevitably face to suffer serious social problems such as the increased number of single elders and accordingly the increasing number of dementia patients. Especially for elders over the age of 80 , the prevalence of dementia becomes very high. Therefore, most people are quite afraid to imagine themselves to suffer dementia in their later years.

In 2012, the Ministry of Health-Welfare (MOWH) in South Korea anticipated that one-million elders (i.e., $10 \%$ of senior citizens over the age of 65) might suffer dementia in 2025, over two-million elders in 2039, and three-million elders in 2050 [1-2]. Also, National Medical Center reported in 2016 that the prevalence of dementia over the age of 85 has been soaring up to $37.5 \%$, which was $7 \%$ higher than 2008 . In addition, it was reported that women (1.9 times), illiterate persons ( $4 \sim 6$ times), poor classes (4.7 times), and single elders (3 4 times) were more likely to suffer dementia. Hence, extremely high cost of 3.4 trillion Korean-won (i.e., 2.8 billion US\$) would have to be spent as social costs for these elders. In Japan, the number of elders occupies 28.7 \% (i.e. 36 million) among 125 million population, in which the dementia patients are expected to be 7 million (i.e. $20 \%$ of elders over 65) in 2025. Also, the Alzheimer Netherland Foundation predicted in 2015 that half a million elders (i.e., $13 \%$ of senior citizens) might suffer dementia in 2040 [3]. 


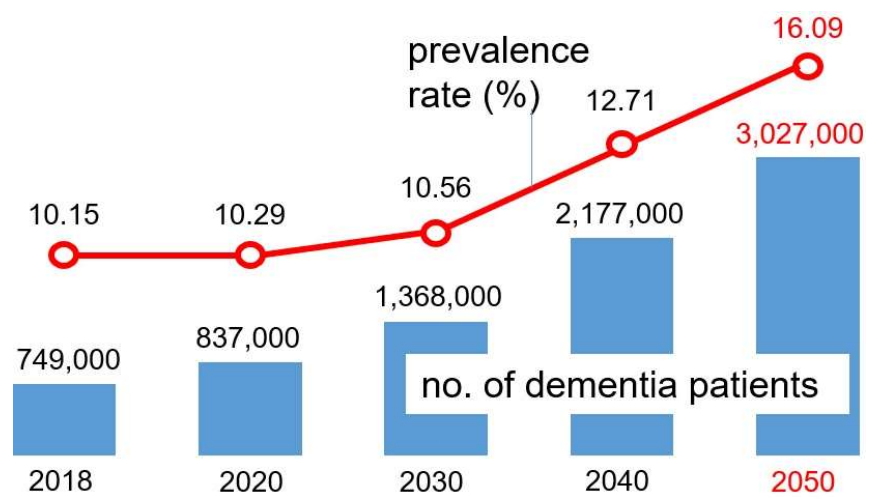

Figure 1. Prospect of senile dementia over the age of 65 (Ministry of Health and Welfare in South Korea 2018).

Most dementia patients are categorized as either Alzheimer patients ( $70 \%)$ or vascular dementia patients ( $\sim 21 \%)$. It is well known that dementia results in gradual brain dysfunction, which deteriorates the psychic function such as memory, language, decision, even emotion [4]. Therefore, dementia destructs not only the dignity of the patients, but also the ordinary lives of their families. Moreover, the cure for dementia has not been developed yet. Hence, the governments and other social well-fare centers have organized elder-care facilities to take care of the serious dementia patients for the sake of their sanctity. However, the current situation in South Korea indicates that the public elder-care hospitals and nursing homes are very short of manpower, i.e., professional nursing nurses, such that only two nurses should serve more than 10 patients in a room who mostly lie down on beds. Recently, COVID-19 has shrunk further down the manpower for dementia patients significantly. Worse is that COVID-19 prevents the families from visiting their parents staying in elder-care hospitals or nursing homes. Even in the death of the patients, their families are forbidden to attend the funerals owing to the fear of virus infection.

In these perilous situations, we believe that it would be a great help and relief to provide a meal monitoring system to nurses and families so that they would be able to see how much food their patients are eating in real-time at least three times a day. It also helps nurses spot the patients who barely eat meals and therefore optimize their capabilities to monitor patients closely [5]. Meanwhile, senile dementia patients sometimes tend to burn their tongues by eating hot soups with no hesitation because they cannot recognize how hot their meals are. This instinctive action might damage their oral hygiene severely. Also, they tend to stop eating food as soon as the meal becomes cold.

Hence, in this paper, we propose a couple of meal monitoring systems using electronic sensor devices in order to acquire the functions to alert the patients the meal temperature and to convey the food diary information of the dementia patients to their nurses and families via wireless transfer in real-time [6-7]. For this purpose, a low-cost Arduino UNO board with a weight sensor is exploited to sense how much food have eaten in every 5 second until the patients finishes their meal. Also, a non-contact optical sensor is utilized to obtain the meal temperature.

Section II describes the proposed meal monitoring systems; first a meal weight monitoring system and then a meal temperature monitoring system. Section III demonstrates the measured results of both monitoring systems. Then, a conclusion is followed.

\section{Meal Monitoring Systems}

In this section, a couple of meal monitoring systems are presented, including a meal weight monitoring system and a meal temperature monitoring system. 


\subsection{Meal Weight Monitoring System}

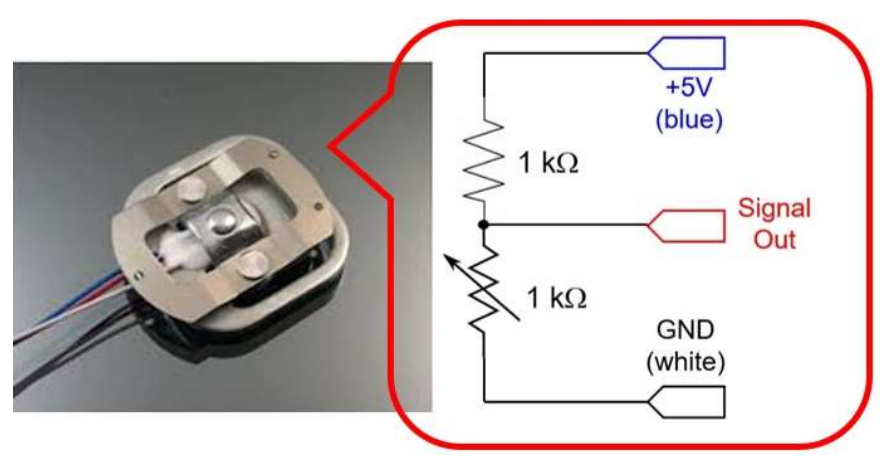

Figure 2. Picture of a three-line load-cell, and its equivalent circuit.

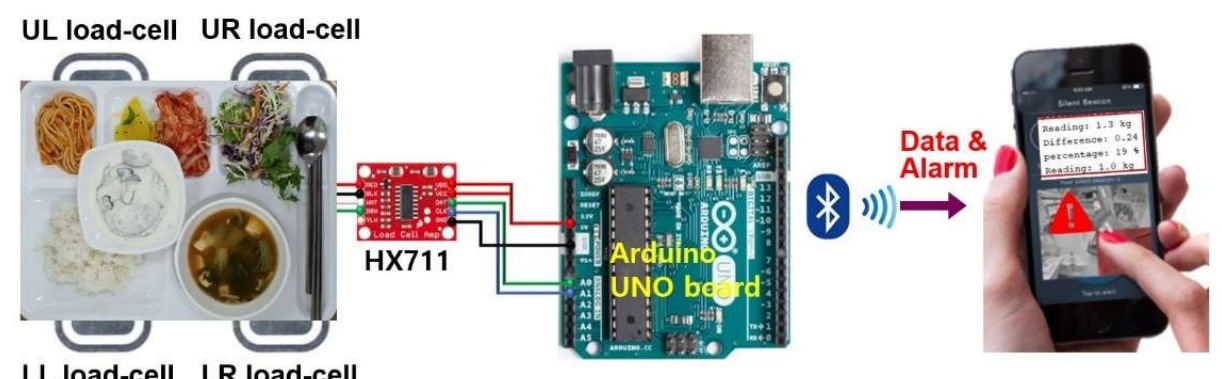

LL load-cell LR load-cell

Figure 3. Conceptual architecture of a meal weight monitoring system.

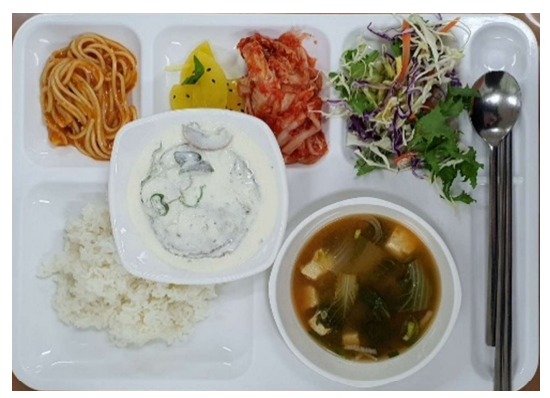

Reading: $-3.5 \mathrm{~kg}$

Reading: $-3.5 \mathrm{~kg}$

Reading: $-3.5 \mathrm{~kg}$

Reading: $-3.5 \mathrm{~kg}$

Reading: $-3.5 \mathrm{~kg}$

Reading: $-3.5 \mathrm{~kg}$

Reading: $-3.5 \mathrm{~kg}$

Reading: $-3.5 \mathrm{~kg}$

Reading: $-3.5 \mathrm{~kg}$

(a)

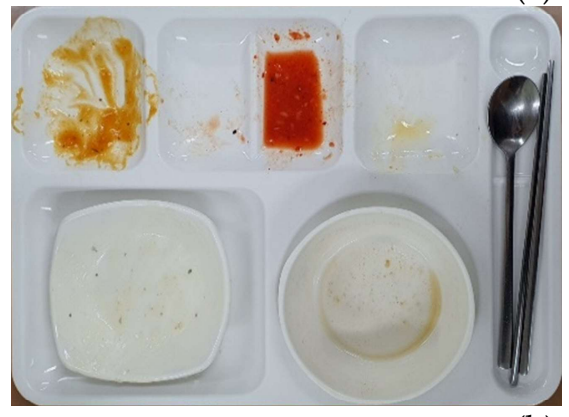

Reading: $-2.0 \mathrm{~kg}$

Reading: $-2.0 \mathrm{~kg}$

Reading: $-2.0 \mathrm{~kg}$

Reading: $-2.0 \mathrm{~kg}$

Reading: $-2.0 \mathrm{~kg}$

Reading: $-2.0 \mathrm{~kg}$

Reading: $-2.0 \mathrm{~kg}$

Reading: $-2.0 \mathrm{~kg}$

Reading: $-2.0 \mathrm{~kg}$

(b)

Figure 4. Meal weight measurements with no calibration factor: (a) initial meal and (b) after the meal time.

The proposed meal weight monitoring system consists of four load-cells, a front-end analog amplifier with an analog-to-digital converter (ADC), and a low-cost Arduino UNO board. Fig. 2 shows the picture of a load-cell (which is limited to the maximum weight of $50 \mathrm{~kg}$ ) and its equivalent circuit, in which it is clearly seen that a weight can change the 
variable resistance of the load-cell, thus generating a different output voltage from a reference voltage. This voltage output is sensed by a following analog amplifier with an ADC (HX711 in this work). Then, a low-cost Arduino UNO board is followed to demonstrate the value of weight and transfer the data wireless over to mobile phones in every 5 second.

Now, the weight of a meal tray for dementia patients is measured by utilizing four load-cells. Each load-cell should be located at the corner of the tray and therefore the corresponding four output voltages can be simultaneously detected by the HX711. Then, the following Arduino UNO board generates the final weight onto a monitor that can be either a small LCD display or a PC monitor (as shown in Fig. 3). In addition, a Bluetooth module compatible with an Arduino board can be utilized to transmit the weight information onto the mobiles phones of both nurses and families, so that they can figure out the amount of food that each patient is taking during the meal time [6]. This would certainly be a great relief to the families and also a crucial information to nurses in charge.

Yet, this configuration may require a critical process called 'initial system calibration' that sets a reference weight for precise measurements. Namely, a calibration factor should be tuned for proper weight information. In this work, the calibration factor is set to 104,000 in the Arduino code. This calibration process typically resets the initial weight to $0 \mathrm{~g}$ for start, and then measures the precise weight of a meal tray. Also, it should be noted that the weight data can be sensitive to the location of four load-cells under a meal tray and therefore, each load-cell should be attached to each corner of the tray. Certainly, a trayshape scale with an identical size would be preferred for this type of weight measurement.

Nonetheless, there exists a merit in this proposed meal weight monitoring system, which is that the relative amount of food left on a tray can be measured even if the reference weight cannot be specified precisely. In other words, even in the cases that nurses forget to tune the calibration factor for precise measurements by any reason, our monitoring system can generate the difference between the original meal weight and the final weight, so that the nurses in charge can easily figure out the amount that each patient have eaten. Fig. 4 shows an example with an uncorrected calibration factor, where the initial weight is read as $-3.5 \mathrm{~kg}$, while the weight of an empty tray when finished is recorded as $-2.0 \mathrm{~kg}$. Although the absolute value of meal weight taken by the patient is not shown, nurses and families can see clearly whether the patient has eaten or not.

\subsection{Meal Temperature Monitoring System}

As aforementioned, the temperature of a meal tray should be measured to protect the patient's tongue and keep oral hygiene because most senile dementia patients cannot recognize how hot their meals are, especially when they are served with hot soups. They swallow hot soups with no hesitation, therefore burning their tongues frequently. Also, most dementia patients tend to stop eating when the meals are getting cold. Hence, we have developed a meal temperature monitoring system using a bipolar junction transistor (BJT) sensor and a low-cost Arduino UNO board, so that the dementia patients can be alert for hot temperature and also the nurses can recognize the requirements to replace cold food with hot ones for some patients. In particular, the alert message can be displayed either on screen or heard through the hearing aid of the patients. 


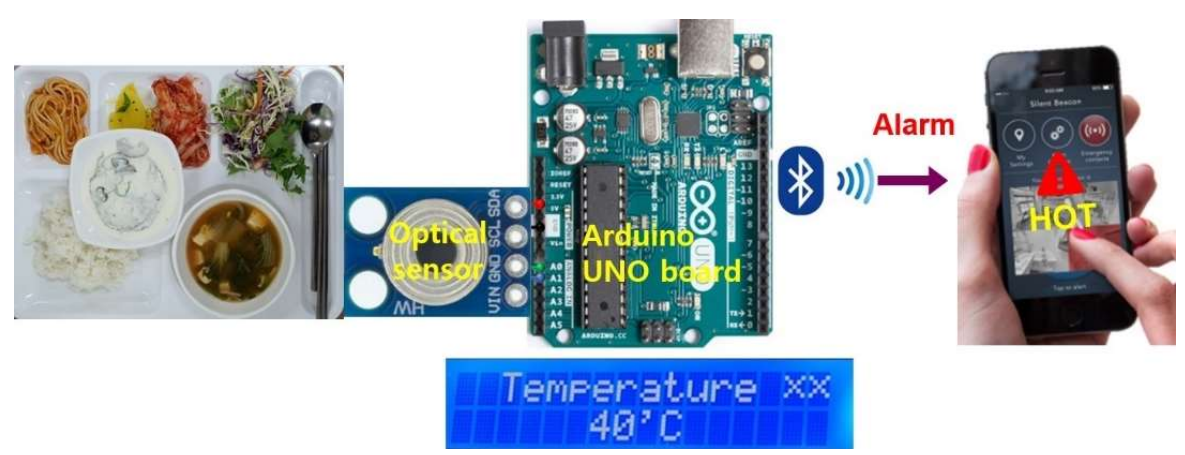

Figure 5. Conceptual architecture of a meal temperature monitoring system.

Fig. 5 shows a conceptual architecture of the proposed meal temperature monitoring system in which a non-contact optical sensor and a LCD display are connected to an Arduino UNO board. Besides, a Bluetooth module compatible with an Arduino board can be utilized to transmit the temperature information onto the mobiles phones of nurses in charge, so that the nurses can serve the patients more effectively, e.g. the cold food can be replaced with hot ones on time for some patients.

In this work, MLX90614 is exploited as an optical sensor that emits infrared lights to targets for temperature measurements in the range between $-70{ }^{\circ} \mathrm{C}$ and $380{ }^{\circ} \mathrm{C}$ with the accuracy of $0.5{ }^{\circ} \mathrm{C}$. The reference temperature was set to $30{ }^{\circ} \mathrm{C}$. Above this reference temperature, any form of alarm can be displayed on the LCD screen. Also, another reference is set to $20^{\circ} \mathrm{C}$, below which it sends an alarm to nurses for food replacement. An example is shown in Fig. 8 and Fig. 9.

\section{Measured Results}

In this section, the practical implementation of the proposed meal monitoring systems is presented along with their measured results.

\subsection{Meal Weight Monitoring System}

Fig. 6 shows an implementation of a weight monitoring module with a single loadcell along with its measured results obtained by pressing a single load-cell with a human hand, where it is clearly seen that the variation of weight is recorded.

Fig. 7 demonstrates a practical implementation of the proposed meal weight monitoring system, where four load-cells are located under the corners of a meal tray. Then, it demonstrates the real-time measurements of the meal weight left on a food tray; (a) the initial weight of a meal tray with food, (b) a meal weight in the middle of meal time, and (c) the final meal weight when finished. In Fig. 7(a), the initial weight of $1.8 \mathrm{~kg}$ is recorded. After a few minutes, Fig. 7(b) records $1.6 \mathrm{~kg}$ (that is $200 \mathrm{~g}$ reduced from the initial weight) and shows the percentage of $11 \%$, indicating that the designate patient has eaten $11 \%$ of the provided meal. Finally, Fig. 7(c) ) presents the final weight of an empty tray, i.e. $1.0 \mathrm{~kg}$ which is already well known to nurses and families. It provides the relative percentage of $43 \%$ which is the ratio of $1.0 \mathrm{~kg}$ to the initial weight of $1.8 \mathrm{~kg}$. Hence, nurses and families can see clearly in real-time how much food the patient has eaten.

In addition, all these acquired information can be continuously transferred wirelessly to the mobile phones of nurses in charge and the patient's family. The left picture in Fig. $7(d)$ shows the screen of a mobile phone with the information of the weight variation from the initial weight in the middle of meal time. The relative difference of weight is also clearly shown. The right picture in Fig. 7(d) corresponds to the case of Fig. 7(c). 

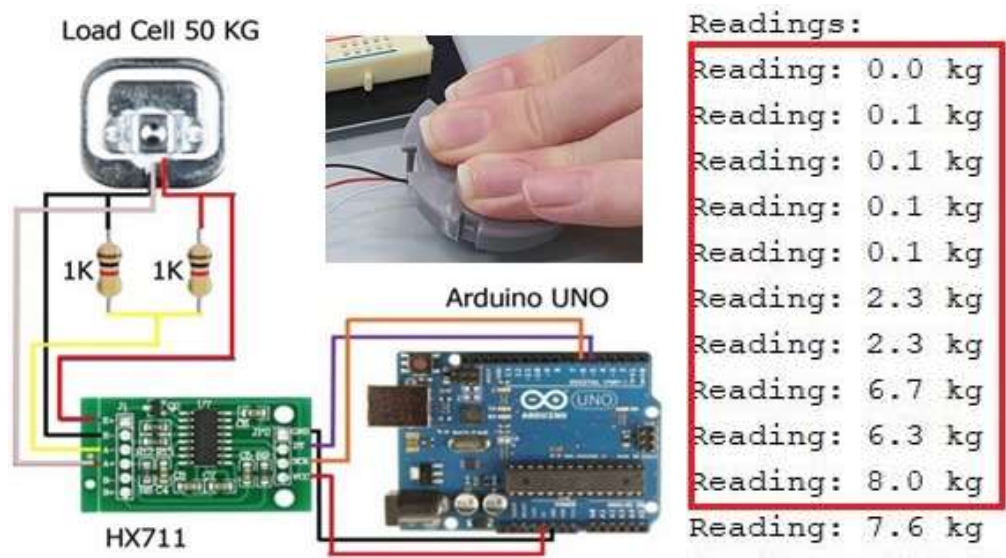

Figure 6. Example of a weight measuring module with a single load-cell.

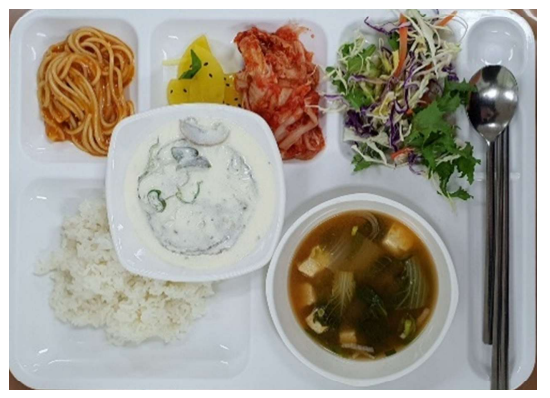

Reading: $1.8 \mathrm{~kg}$

Difference: -0.00

percentage: 0 \&

Reading: $1.8 \mathrm{~kg}$

Difference: 0.00

percentage: 0 \&

Reading: $1.8 \mathrm{~kg}$

Difference: 0.00

percentage: $0 \&$

(a)

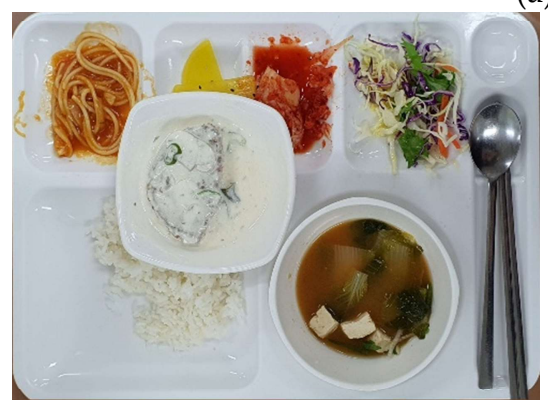

difference: $0.2 \mathrm{~kg}$ percentage: 11 \% reading: $1.6 \mathrm{~kg}$ difference: $0.2 \mathrm{~kg}$ percentage: 11 \% reading: $1.6 \mathrm{~kg}$ difference: $0.2 \mathrm{~kg}$ percentage: 11 \% reading: $1.6 \mathrm{~kg}$ difference: $0.2 \mathrm{~kg}$ percentage: 11 \%

(b)

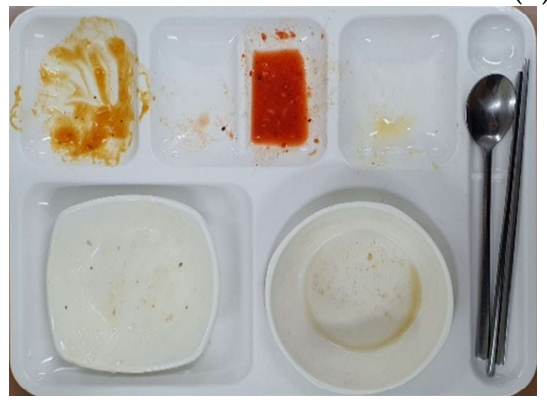

difference: $0.8 \mathrm{~kg}$ percentage: $43 \%$ reading: $1.0 \mathrm{~kg}$ difference: $0.8 \mathrm{~kg}$ percentage: $43 \%$ reading: $1.0 \mathrm{~kg}$ difference: $0.8 \mathrm{~kg}$ percentage: 43 \& reading: $1.0 \mathrm{~kg}$ difference: $0.8 \mathrm{~kg}$ percentage: 43 \%

(c) 


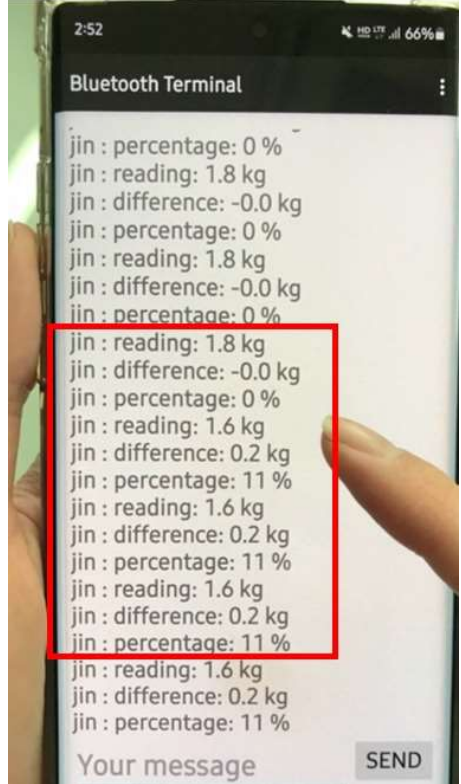

(d)

Figure 7. Meal weight measurements with a corrected calibration factor: (a) initial meal, (b) meal in the middle, (c) after the meal time, and (d) wireless transfer via Bluetooth.

\subsection{Meal Temperature Monitoring System}

In order to demonstrate an example of temperature measurements, we exploit a noncontact optical sensor (MLX90614) and an Arduino UNO board with a LCD display. Here, the reference temperature was set to $30{ }^{\circ} \mathrm{C}$. Fig. 8 demonstrates the measured results of the meal temperature monitoring system for three different temperatures. First, Fig. 8(a) shows the temperature of $40{ }^{\circ} \mathrm{C}$, which is high above the reference. Therefore, it shows a form of alarm (XX mark in this work) on a LCD screen for hot temperature.

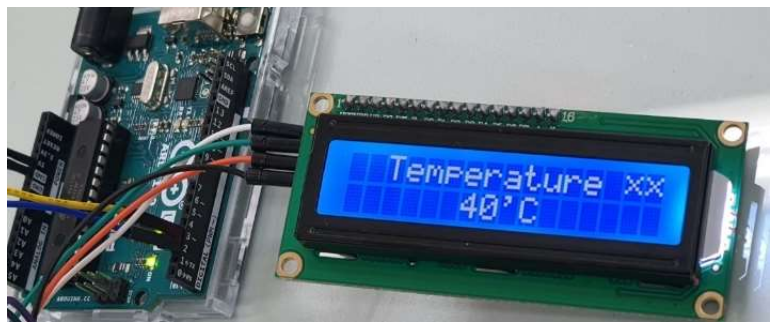

(a)

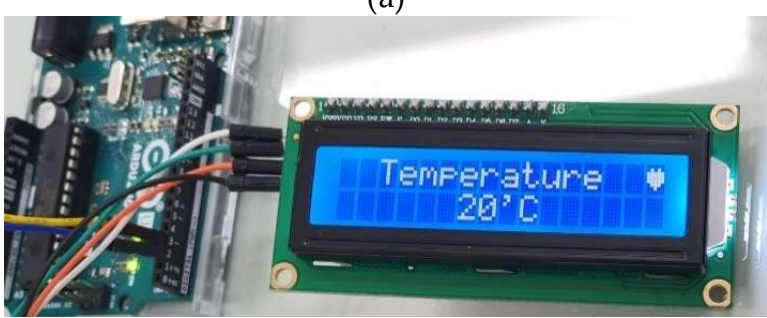

(b) 


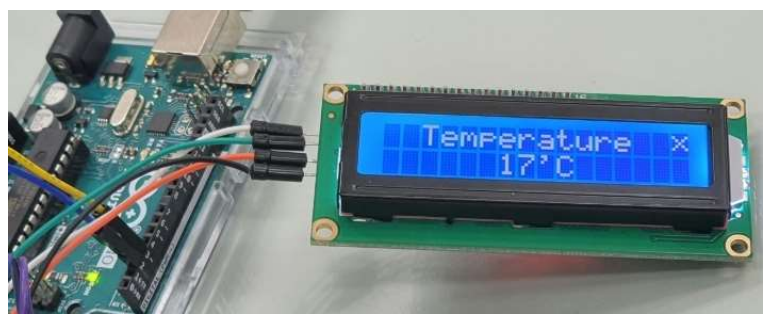

(c)

Fig. 8. Meal temperature measurements by using a LCD display: (a) above (XX), (b) normal (heart), and (c) below $(\mathrm{X})$ the reference temperature.

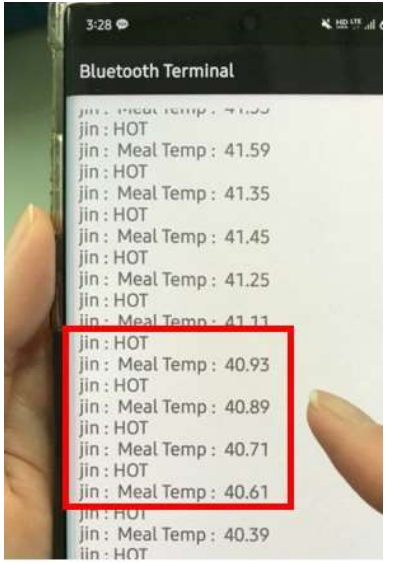

(a)

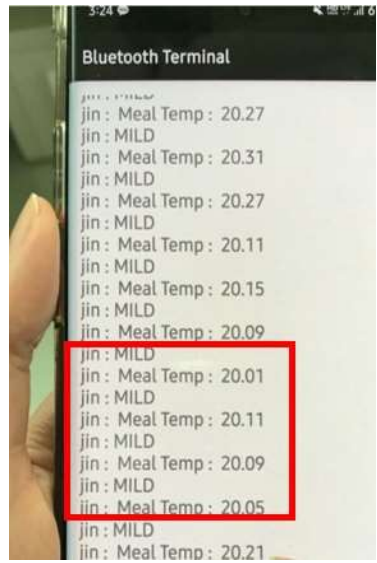

(b)

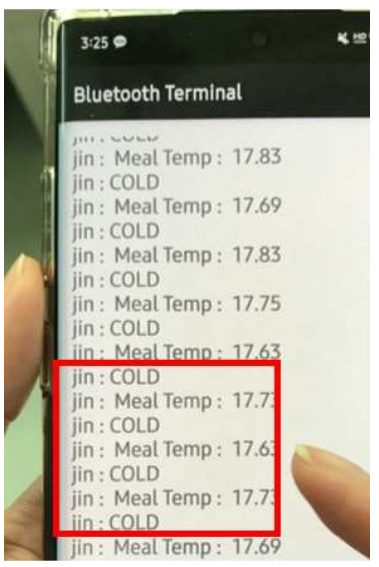

(c)

Figure 9. Wireless transfer over a mobile phone by using Bluetooth network: (a) hot (over $40{ }^{\circ} \mathrm{C}$ ), (b) mild (in between), and (c) cold (below $20^{\circ} \mathrm{C}$ ) temperatures.

Second, Fig. 8(b) demonstrates the mild temperature of $20^{\circ} \mathrm{C}$ and no alarm is turned on yet, rather showing a heart sign in this work. Third, when the meal temperature below $20{ }^{\circ} \mathrm{C}$ is detected, the monitoring system sends an alarm signal to nurses so that the cold meal can be replaced with hot ones if necessary. Third, Fig. 8(c) depicts the temperature of $17{ }^{\circ} \mathrm{C}$ which is below the other reference of $20^{\circ} \mathrm{C}$. Then, a $\mathrm{X}$ mark is displayed on the LCD screen as a token of alarm. The size of an LCD display utilized in this work can be replaced with a much larger one for senile dementia patients in order to recognize the alarm vividly.

Fig. 9 presents the wireless transfer of the temperature information onto the mobile phone via a Bluetooth network, so that nurses can easily figure out the necessity to replace cold food with hot ones. Also, families can see the state of food temperature served in elder-care hospitals or nursing homes in real-time.

\section{Conclusion}

We have implemented a couple of meal monitoring systems for senile dementia patients by utilizing electronic sensors together with a low-cost Arduino UNO board in order to measure the meal weight left on a food tray in real-time during when the patients are eating and also to measure the temperature of a hot meal. Thereby, the amount of food taken by the dementia patients are easily recorded and even transferred wirelessly via either Bluetooth or WiFi networks onto the mobile phones of both the nurses in charge and the families of the patients instantly. Also, the hot temperature of the served meal is displayed on screen so that the patients can be alert to avoid the burning of their tongues and to maintain their oral hygiene. Hence, it can be concluded that the proposed meal monitoring systems will help the senile dementia patients intake more food and thus enlengthen their precious lives in sanctity. Also, they can provide a great help and relief not 
only for the families of senile dementia patients, but also for nurses in elder-care hospitals and nursing homes.

Author Contributions: Conceptualization and methodology, S. M. Park.; coding and validation, J. Joo, Y. Hu, H. Hwang, Y. Jeon, J. Jung; writing-original draft preparation, S. M. Park.; writingreview and editing, J. Joo, S. M. Park; supervision, project administration, funding acquisition, S. M. Park. All authors have read and agreed to the published version of the manuscript..

Funding: This work was supported by the National Research Foundation of Korea (NRF) grant funded by the Korea government (MSIT) (No. 2020R1A2C1008879).

Acknowledgments: Authors would like to thank Prof. Duk Yoo Jung and Prof. Juh Hyun Shin in College of Nursing at Ewha Womans University for their clinical advice.

\section{References}

1. Cho, H.; Ko, Z. (2012). Current State of Senile Dementia and Improvement of the Long Term Care Insurance for Elderly People. J. of the Korea Academia-Industrial Cooperation Society, 13(12), 5816-5825.

2. Yoon, D; Joo, J. E.; Park, S. M. (2021). Mirrored Current-Conveyor Transimpedance Amplifier for Home Monitoring LiDAR Sensors. IEEE Sensors Journal, 21(5), 5589-5597.

3. Boer, B. D.; Hamers, J.; Beerens, H.; Zwakhalen, S.; Tan, F.; Verbeek, H. (2015). Living at the farm, innovative nursing home care for people with dementia - study protocol of an observational longitudinal study. BMD Geriatrics, 15(1).

4. Alexandra, K.; Junior, C.; Fernando, C.; Alexandre, D.; Gregory, B.; Pierre-David, P.; Francois, B.; Renaud, D.; Frans, V.; Pauline, A.; Philippe, R. (2015). Validation of an Automatic Video Monitoring System for the Detection of Instrument Activities of Daily Living in Dementia Patients. J. of Alzheimer's Disease, 44(2), 675-685.

5. Jordan, S.; Gabe-Walters, M. E.; Watkins, A.; Humphreys, I.; Newson, L.; Snelgrove, S.; Dennis, M. S. (2015). Nurse-Led Medicines' Monitoring for Patients with Dementia in Care Homes: A Pragmatic Cohort Stepped Wedge Cluster Randomized Trial. PLOS ONE, doi.org/10.1371/journal.pone.0140203.

6. Kim, S. -H.; He, Y.; Lee, E. -H.; .Kim, J. -H.; Park, S. M. (2017). Portable Fluormeter for Cyanobacterial Detection. IEEE Sensors Journal, 17(8), 2377-2384.

7. He, Y.; Kim, J. -H.; Park, S. M. (2020). A CMOS Read-Out IC for Cyanobacteria Detection With 40 nApp Sensitivity and 45-dB Dynamic Range. IEEE Sensors Journal, 20(8), 4283-4289. 